\title{
Are human biomarker studies always more valuable than animal ones?
}

\section{Opinion}

In biomarker discovery study, are experiments with more human clinical samples always more valuable than the ones with fewer animal model samples?

Biomarker study is to search for the changes associated with the disease. The key words are changes and association. Since we are looking for changes, we want to look at the place where there are full of changes. Theoretically urine accumulates more changes than blood, where changes are minimized by homeostasis mechanisms of the body. ${ }^{1}$ The problem of looking for changes in urine is there are too many changes. The difficulty is not to find changes, but to find the definitive association with the disease. When there are too many factors can affect urine, using big data strategy to sort out all the factors known and unknown, and to find association with disease is time and money consuming, even unfeasible most of the time. We can handle a lot more experiments and a lot more data now, but not that much yet. Instead, limiting the factors to minimal can be more effective and realistic in finding the true association.

Studying with clinical samples has always been considered more superior than the study with animal model. They are easier accepted by famous journal in the field. No doubt it holds the ultimate pass to the bedside. But in discovery phase, we can let the disease in animal develop without medication which is impossible to do clinically. We can control what the animals to eat to drink and where to live, which is almost impossible to do in real life. If those factors do not affect the potential biomarkers at all, it is fine to search with clinical samples. But even changes of physiological condition and medication ${ }^{2,3}$ will be reflected in urine. Studies using animal model even with fewer sample size may establish the association with the disease better than studies with even more clinical samples. It simply has few factor involved.

People always argue that animal models are not the real disease. They are not real indeed. But they are somewhat similar to the disease. For biomarker study, we don't need to be exactly the same. It would be great as long as we find the potential biomarkers associated with the pathophysiological features in the model that are similar to the disease. And we can always try different models for the same disease to cover the features that previous model missed.

\author{
Volume 2 Issue I - 2015 \\ Youhe Gao \\ Department of Biochemistry and Molecular Biology, Beijing \\ Normal University, China
}

\begin{abstract}
Correspondence: Youhe Gao, Department of Biochemistry and Molecular Biology, Beijing Normal University, Gene Engineering and Biotechnology Beijing Key Laboratory, Beijing, I00875, P. R. of China, Email youhegao@I63.com
\end{abstract}

Received: January 16, 2015 | Published: January 21, 2015

Of course, animal model and human clinical samples are both necessary in biomarker studies. But I hope that both editors and reviewers can give a second thought when evaluating manuscripts in discovery phase, to see which studies have fewer factors involved and which ones give the more definitive ASSOCIATION with the disease instead of to merely see which ones have the bigger number of samples and if the results are from clinical samples.

\section{Acknowledgements}

None.

\section{Conflict of interest}

The author declares no conflict of interest.

\section{References}

1. Gao Y. Urine - an untapped goldmine for biomarker discovery? Sci China Life Sci. 2013;56(12):1145-1146.

2. Li M, Zhao M, Gao Y. Changes of proteins induced by anticoagulants can be more sensitively detected in urine than in plasma. Sci China Life Sci. 2014;57(7):649-656

3. Li X, Zhao M, Li M, et al. Effects of Three Commonly-used Diuretics on the Urinary Proteome. Genomics Proteomics Bioinformatics. 2014;12(3):120-126. 